\title{
Iterated remainder operator, tests for multiple convergence of series, and solutions of difference equations
}

Janusz Migda*

\section{"Correspondence:}

migda@amu.edu.pl

Faculty of Mathematics and

Computer Science, A. Mickiewicz

University, Umultowska 87, Poznań,

61-614, Poland

\begin{abstract}
We establish some properties of iterations of the remainder operator which assigns to any convergent series the sequence of its remainders. Moreover, we introduce the spaces of multiple absolute summable sequences. We also present some tests for multiple absolute convergence of series. These tests extend the well-known classical tests for absolute convergence of series. For example we generalize the Raabe, Gauss, and Bertrand tests. Next we present some applications of our results to the study of asymptotic properties of solutions of difference equations. We use the spaces of multiple absolute summable sequences as the measure of approximation.
\end{abstract}

MSC: $39 A 10$

Keywords: remainder operator; Raabe's test; Gauss's test; Bertrand's test; difference equation; prescribed asymptotic behavior; asymptotically polynomial solution

\section{Introduction}

Let $r$ denote the operator which assigns to any convergent series the sequence of its remainders. The purpose of this paper is to study the basic properties of iterations $r^{m}$ of $r$ and apply these results to the study of asymptotic properties of solutions of difference equations. We also study the spaces of multiple absolute summable sequences. Moreover, we obtain extensions of some classical tests for absolute convergence of series.

The paper is organized as follows. In Section 2, we introduce our notation and terminology. In Section 3, we define the iterations of the remainder operator and the spaces $\mathrm{S}(\mathrm{m})$ of $m$-times summable sequences. Moreover, we establish some basic properties of $r^{m}$. For example, we show that

$$
\Delta^{m} r^{m} x=(-1)^{m} x \quad \text { and } \quad r^{m} \Delta^{m} z=(-1)^{m} z
$$

for any sequence $x \in \mathrm{S}(m)$ and any sequence $z$ convergent to zero. This means that the operator $(-1)^{m} r^{m}$ is inverse to the restriction $\Delta^{m} \mid Z$ where $Z$ denotes the space of all convergent to zero sequences.

In Section 4 , we introduce the spaces $\mathrm{A}(m)$ of absolutely $m$-times summable sequences and establish the relationships between $\mathrm{A}(m)$ and the spaces $\mathrm{O}\left(n^{s}\right)$. We also extend some

\section{Springer}

(02014 Migda; licensee Springer. This is an Open Access article distributed under the terms of the Creative Commons Attribution License (http://creativecommons.org/licenses/by/2.0), which permits unrestricted use, distribution, and reproduction in any medium, provided the original work is properly cited. 
classical tests for absolute convergence of series. For example, the Raabe test states that

$$
\text { if } \liminf n\left(\frac{\left|a_{n}\right|}{\left|a_{n+1}\right|}-1\right)>1, \quad \text { then } \sum_{n=1}^{\infty}\left|a_{n}\right|<\infty
$$

In Lemma 4.5 we show that

$$
\text { if } \liminf n\left(\frac{\left|a_{n}\right|}{\left|a_{n+1}\right|}-1\right)>2, \quad \text { then } \sum_{n=1}^{\infty} \sum_{k=n}^{\infty}\left|a_{k}\right|<\infty
$$

and, more generally,

$$
\text { if } \liminf n\left(\frac{\left|a_{n}\right|}{\left|a_{n+1}\right|}-1\right)>m, \quad \text { then } \sum_{i_{1}=1}^{\infty} \sum_{i_{2}=i_{1}}^{\infty} \ldots \sum_{i_{m}=i_{m-1}}^{\infty}\left|a_{i_{m}}\right|<\infty \text {. }
$$

On the other hand,

$$
\text { if } n\left(\frac{\left|a_{n}\right|}{\left|a_{n+1}\right|}-1\right) \leq m \quad \text { for large } n, \quad \text { then } \sum_{i_{1}=1}^{\infty} \sum_{i_{2}=i_{1}}^{\infty} \ldots \sum_{i_{m}=i_{m-1}}^{\infty}\left|a_{i_{m}}\right|=\infty
$$

Similarly in Lemma 4.4 we show that

$$
\limsup \frac{\log \left|a_{n}\right|}{\log n}<-m \text { implies } \sum_{i_{1}=1}^{\infty} \sum_{i_{2}=i_{1}}^{\infty} \ldots \sum_{i_{m}=i_{m-1}}^{\infty}\left|a_{i_{m}}\right|<\infty
$$

and

$$
\frac{\log \left|a_{n}\right|}{\log n} \geq-m \quad \text { for large } n \quad \text { implies } \quad \sum_{i_{1}=1}^{\infty} \sum_{i_{2}=i_{1}}^{\infty} \cdots \sum_{i_{m}=i_{m-1}}^{\infty}\left|a_{i_{m}}\right|=\infty .
$$

For $x \in \mathrm{S}(m)$, by definition, we have

$$
r^{m}(x)(n)=\sum_{i_{1}=n}^{\infty} \sum_{i_{2}=i_{1}}^{\infty} \cdots \sum_{i_{m}=i_{m-1}}^{\infty} x_{i_{m}}
$$

If $|x| \in \mathrm{S}(m)$, i.e., $x \in \mathrm{A}(m)$, then $x \in \mathrm{S}(m)$ and

$$
r^{m}(x)(n)=\sum_{k=0}^{\infty}\left(\begin{array}{c}
m-1+k \\
m-1
\end{array}\right) x_{n+k}=\sum_{i=n}^{\infty}\left(\begin{array}{c}
m+i-n-1 \\
m-1
\end{array}\right) x_{i} .
$$

We use (3) to obtain basic properties of the operator $r^{m}$. At the end of Section 4, using all possible $\Delta^{p} \mathrm{~A}(m)$ and $r^{p} \mathrm{~A}(m)$, we obtain an infinite stratification of the space of all sequences convergent to zero. This stratification induces a substratification of $\mathrm{A}(m)$ for any $m$.

In Section 5, we present some applications of our results to the study of asymptotic properties of solutions of difference equations. We use the spaces $\mathrm{A}(p)$ as the measure of approximation. For example, we apply our results and fixed point theorems to the study of 
solutions with prescribed asymptotic behavior. More precisely, using the Schauder fixed point theorem and the Knaster-Tarski fixed point theorem, we establish conditions under which for a given sequence $b$ and a solution $y$ of the equation $\Delta^{m} y=b$ there exists a solution $x$ of the equation

$$
\Delta^{m} x_{n}=a_{n} f\left(x_{\sigma(n)}\right)+b_{n}
$$

such that

$$
x \in y+\mathrm{A}(p) .
$$

We also show that if $a, b \in \mathrm{A}(m+p)$ and $x$ is a solution of (4) such that the sequence $\left(f\left(x_{n}\right)\right)$ is bounded, then

$$
x \in \operatorname{Ker} \Delta^{m}+\mathrm{A}(p) .
$$

Hence $x$ is asymptotically polynomial. The equality

$$
\Delta^{m}\left((-1)^{m} r^{m} x\right)=x
$$

for $x \in \mathrm{S}(m)$, which is a consequence of (1), plays a crucial role in the application of fixed point theorems to the study of solutions of difference equations. The value $r^{m} x$ is used, mainly implicitly, in the study of solutions with prescribed asymptotic behavior. In some papers the multiple sums (2) appear explicitly; see for example [1-4] or [5]. This paper is a continuation of the papers $[4,6]$ and [7]. Our studies were inspired by the papers [8-12] and the papers [13-17], and [18].

Some applications of our results to the study of asymptotic properties of solutions of nonautonomous difference equations are presented in [19].

\section{Notation and terminology}

Let $\mathbb{N}, \mathbb{Z}, \mathbb{R}$ denote the set of positive integers, the set of all integers and the set of real numbers, respectively. The space of all sequences $x: \mathbb{N} \rightarrow \mathbb{R}$ we denote by SQ.

If $p, k \in \mathbb{Z}, p \leq k$, then $\mathbb{N}(p), \mathbb{N}(p, k)$ denote the sets defined by

$$
\mathbb{N}(p)=\{p, p+1, \ldots\}, \quad \mathbb{N}(p, k)=\{p, p+1, \ldots, k\} .
$$

If $x, y$ in $\mathrm{SQ}$, then $x y$ denotes the sequence defined by pointwise multiplication

$$
x y(n)=x_{n} y_{n} .
$$

Moreover, $|x|$ denotes the sequence defined by $|x|(n)=\left|x_{n}\right|$ for every $n$.

We use the symbols 'big O' and 'small o' in the usual sense but for $a \in$ SQ we also regard $\mathrm{o}(a)$ and $\mathrm{O}(a)$ as subspaces of SQ. More precisely, let

$$
\mathrm{o}(1)=\{x \in \mathrm{SQ}: x \text { is convergent to zero }\}, \quad \mathrm{O}(1)=\{x \in \mathrm{SQ}: x \text { is bounded }\}
$$


and for $a \in$ SQ let

$$
\mathrm{o}(a)=a \mathrm{o}(1)=\{a x: x \in \mathrm{o}(1)\}, \quad \mathrm{O}(a)=a \mathrm{O}(1)=\{a x: x \in \mathrm{O}(1)\} .
$$

For $m \in \mathbb{N}(0)$ we define

$$
\operatorname{Pol}(m-1)=\operatorname{Ker} \Delta^{m}=\left\{x \in \mathrm{SQ}: \Delta^{m} x=0\right\} .
$$

Then $\operatorname{Pol}(m-1)$ is the space of all polynomial sequences of degree less than $m$. For $m \in$ $\mathbb{N}(0), k \in \mathbb{N}$ we define numbers $s_{k}^{m}$ by

$$
s_{k}^{m}=\left(\begin{array}{c}
m+k-1 \\
m
\end{array}\right)=\frac{k(k+1) \cdots(k+m-1)}{m !} .
$$

For $p \in \mathbb{N}$ we define

$$
\operatorname{Fin}(p)=\left\{x \in \mathrm{SQ}: x_{n}=0 \text { for } n \geq p\right\}
$$

We say that a subset $U$ of a metric space $X$ is a uniform neighborhood of a subset $Y$ of $X$ if there exists a positive number $\varepsilon$ such that

$$
\bigcup_{y \in Y} \mathrm{~B}(y, \varepsilon) \subset U
$$

where $\mathrm{B}(y, \varepsilon)$ denotes an open ball of radius $\varepsilon$ about $y$.

A sequence $x \in \mathrm{SQ}$ is called nonoscillatory if $x_{n} x_{n+1} \geq 0$ for large $n$.

Assume $f: \mathbb{R} \rightarrow \mathbb{R}$. We say that a sequence $x \in \mathrm{SQ}$ is $f$-bounded if the sequence $f \circ x$ is bounded. Note that $x \circ \sigma$ is $f$-bounded for any $f$-bounded sequence $x$ and any sequence $\sigma: \mathbb{N} \rightarrow \mathbb{N}$.

Example 2.1 If $f(t)=e^{t}$, then $f$-boundedness of a sequence $x$ is equivalent to the boundedness above of $x$. Assume $f(t)=t^{-1}$ for $t \neq 0, f(0)=3$ and let $L(x)$ denote the set of limit points of a given sequence $x$. Then $f$-boundedness of $x$ is equivalent to the condition $0 \notin L(x)$.

\section{Iterated remainder operator}

In this section we introduce the remainder operator $r$, the spaces $S(m)$ of $m$-times summable sequences and iterations $r^{m}: \mathrm{S}(m) \rightarrow \mathrm{S}(0)$. Next in Lemma 3.1 we establish some basic properties of $r^{m}$ and the relationships between $r^{m}$ and $\Delta^{m}$. Let

$$
\begin{aligned}
& \mathrm{S}(0)=\mathrm{o}(1)=\left\{x \in \mathrm{SQ}: \lim x_{n}=0\right\}, \\
& \mathrm{S}(1)=\left\{x \in \mathrm{SQ}: \text { the series } \sum x_{n} \text { is convergent }\right\} .
\end{aligned}
$$

For $x \in \mathrm{S}(1)$, we define the sequence $r(x)$ by the formula

$$
r(x)(n)=\sum_{j=n}^{\infty} x_{j} .
$$


Then $r(x) \in \mathrm{S}(0)$ and we obtain the remainder operator

$$
r: \mathrm{S}(1) \rightarrow \mathrm{S}(0)
$$

For $m \in \mathbb{N}$ we define, by induction, the linear space $\mathrm{S}(m+1)$ and the linear operator $r^{m+1}$ : $\mathrm{S}(m+1) \rightarrow \mathrm{S}(0)$ by

$$
\mathrm{S}(m+1)=\left\{x \in \mathrm{S}(m): r^{m}(x) \in \mathrm{S}(1)\right\}, \quad r^{m+1}(x)=r\left(r^{m}(x)\right) .
$$

The value $r^{m}(x)(n)$ we denote also by $r_{n}^{m}(x)$ or simply $r_{n}^{m} x$.

Lemma 3.1 Assume $x, y \in \mathrm{SQ}, m, k \in \mathbb{N}, p \in \mathbb{N}(0)$ and $q \in \mathbb{N}(0, m-1)$. Then

(01) if $|x| \in \mathrm{S}(m)$, then $x \in \mathrm{S}(m)$ and $\left|r^{m} x\right| \leq r^{m}|x|$,

(02) $|x| \in \mathrm{S}(m)$ if and only if $\sum_{n=1}^{\infty} s_{n}^{m-1}\left|x_{n}\right|<\infty$,

(03) $|x| \in \mathrm{S}(m)$ if and only if $\sum_{n=1}^{\infty} n^{m-1}\left|x_{n}\right|<\infty$,

(04) $|x| \in \mathrm{S}(m)$ if and only if $\mathrm{O}(x) \subset \mathrm{S}(m)$,

(05) if $|x| \in \mathrm{S}(m)$, then $r_{n}^{m} x=s_{1}^{m-1} x_{n}+s_{2}^{m-1} x_{n+1}+s_{3}^{m-1} x_{n+2}+\cdots$,

(06) if $|x| \in \mathrm{S}(m)$, then $r_{k}^{m}|x| \leq \sum_{n=k}^{\infty} n^{m-1}\left|x_{n}\right|$,

(07) if $x \in \mathrm{S}(m)$, then $\Delta^{m} r^{m} x=(-1)^{m} x$,

(08) if $x=\mathrm{o}(1)$, then $\Delta^{m} x \in \mathrm{S}(m)$ and $r^{m} \Delta^{m} x=(-1)^{m} x$,

(09) $\Delta^{m} \mathrm{~S}(0)=\mathrm{S}(m), r^{m} \mathrm{~S}(m)=\mathrm{S}(0)$,

(10) $\Delta^{p} \mathrm{~S}(m)=\mathrm{S}(m+p), r^{p} \mathrm{~S}(m+p)=\mathrm{S}(m)$,

(11) if $x, y \in \mathrm{S}(m)$ and $x_{n} \leq y_{n}$ for $n \geq p$, then

$$
r_{n}^{m} x \leq r_{n}^{m} y \quad \text { for } n \geq p
$$

(12) if $x \in \mathrm{S}(m)$ and $y_{n}=x_{n}$ for $n \geq p$, then $y \in \mathrm{S}(m)$ and

$$
r_{n}^{m} y=r_{n}^{m} x \quad \text { for } n \geq p
$$

(13) if $y \in \mathrm{S}(m)$ and $0 \leq x \leq y$, then $x \in \mathrm{S}(m)$,

(14) if $|x| \in \mathrm{S}(m)$ and $y$ is bounded, then $y x \in \mathrm{S}(m)$ and

$$
\left|r^{m}(y x)\right| \leq|y| r^{m}|x|
$$

(15) if $|y x| \in \mathrm{S}(m),|y|$ is nondecreasing and positive, then $|x| \in \mathrm{S}(m)$,

$$
|y| r^{m}|x| \leq r^{m}|y x| \quad \text { and } \quad \Delta^{q} r^{m} x=\mathrm{o}\left(n^{-q} y^{-1}\right)
$$

(16) if $t \in(-\infty, 0]$ and $\sum_{n=1}^{\infty} n^{m-t-1}\left|x_{n}\right|<\infty$, then $\Delta^{q} r^{m} x=\mathrm{o}\left(n^{t-q}\right)$.

Proof Assertion (01) is proved in Lemma 1 of [6]. Assertion (02) is proved in Lemma 2 of [6]. (03) is proved in Lemma 3 of [6]. Since $|x| \in O(x)$ we see that (04) is a consequence of (03). (05) is proved in Lemma 2 of [6]. Assertion (06) follows from (05). Assertion (07) is proved in Lemma 5 of [6]. Assertion (08) follows from Lemma 6 of [6]. Assertion (09) is an easy consequence of (08). Assertion (10) is a consequence of (09) and (08). Assertion (11) is obvious for $m=1$. For $m>1$ it can easily be proved by induction. Assertion (12) is 
an easy consequence of (11). Assertion (13) is well known for $m=1$. Assume it is true for certain $m \geq 1$. Let $0 \leq x \leq y$ and $y \in \mathrm{S}(m+1)$. Then $y \in \mathrm{S}(m)$ and, by assumption, $x \in \mathrm{S}(m)$. Moreover, using (11) we have

$$
0 \leq r^{m} x \leq r^{m} y
$$

and, by (10), $r^{m} y \in \mathrm{S}(1)$. Hence $r^{m} x \in \mathrm{S}(1)$ and we obtain $x \in \mathrm{S}(m+1)$. Assertion (14) follows from (03) and Lemma 4 of [6]. Assume $|y|$ is nondecreasing and positive and $|y x| \in \mathrm{S}(m)$. Then the sequence $y^{-1}$ is bounded and using (04) we have $|x|=|y|^{-1}|y x| \in \mathrm{S}(m)$. Moreover, using (05), we have

$$
\begin{aligned}
\left|y_{n}\right| r_{n}^{m}|x| & =\left|y_{n}\right| s_{1}^{m-1}\left|x_{n}\right|+\left|y_{n}\right| s_{2}^{m-1}\left|x_{n+1}\right|+\left|y_{n}\right| s_{3}^{m-1}\left|x_{n+2}\right|+\cdots \\
& \leq s_{1}^{m-1}\left|y_{n} x_{n}\right|+s_{2}^{m-1}\left|y_{n+1} x_{n+1}\right|+s_{3}^{m-1}\left|y_{n+2} x_{n+2}\right|+\cdots=r_{n}^{m}|y x|
\end{aligned}
$$

for any $n$. Hence $|y| r^{m}|x| \leq r^{m}|y x|$. Using (01), we obtain

$$
|y|\left|r^{m} x\right| \leq|y| r^{m}|x| \leq r^{m}|y x|=\mathrm{o}(1) \quad \text { and } \quad r^{m} x=\mathrm{o}\left(y^{-1}\right) .
$$

By (03), $\left|y n^{q} x\right| \in \mathrm{S}(m-q)$. Hence, replacing $y$ by $n^{q} y$ and $m$ by $m-q$ in (5), we obtain $r^{m-q} x=\mathrm{o}\left(n^{-q} y^{-1}\right)$. Therefore

$$
\Delta^{q} r^{m} x=\Delta^{q} r^{q} r^{m-q} x=(-1)^{q} r^{m-q} x=\mathrm{o}\left(n^{-q} y^{-1}\right)
$$

and we obtain (15). Using (03) and taking $y_{n}=n^{-t}$ in (15) we obtain (16).

Remark 3.1 For $x \in \mathrm{S}(m)$ and $n \in \mathbb{N}$, by definition of $r^{m}$, we have

$$
r_{n}^{m} x=\sum_{i_{1}=n}^{\infty} \sum_{i_{2}=i_{1}}^{\infty} \ldots \sum_{i_{m}=i_{m-1}}^{\infty} x_{i_{m}} .
$$

Moreover, if $|x| \in \mathrm{S}(m)$, then, by Lemma 3.1(05), we have

$$
r_{n}^{m} x=\sum_{k=0}^{\infty}\left(\begin{array}{c}
m-1+k \\
m-1
\end{array}\right) x_{n+k}
$$

Remark 3.2 By Lemma 3.1(07) and (08) the restriction $\Delta^{m} \mid S(0): S(0) \rightarrow S(m)$ is a bijection with inverse $(-1)^{m} r^{m}$.

Remark 3.3 If $X$ is a linear subspace of $\mathrm{S}(0)$ and $Y$ is a linear subspace of $\mathrm{S}(m)$, then, using Lemma 3.1(08) and (07), we have

$$
r^{m} \Delta^{m} X=X, \quad \Delta^{m} r^{m} Y=Y .
$$

For any $m \in \mathbb{N}$ we have $\mathrm{S}(m) \subset \mathrm{S}(0)=\mathrm{o}(1)=\mathrm{o}\left(n^{0}\right)$. In the following example we show that for any $m \in \mathbb{N}$ and any $\varepsilon>0$ there exists a sequence $x$ such that

$$
x \in \mathrm{S}(m) \backslash \mathrm{O}\left(n^{-\varepsilon}\right)
$$

and, moreover, $\lim _{n \rightarrow \infty} n^{\varepsilon}\left|x_{n}\right|=\infty$. 
Example 3.1 Let $m \in \mathbb{N}$ and $\varepsilon>0$. Choose $\delta \in(0, \varepsilon)$. Let $y_{n}=(-1)^{n} n^{-\delta}$ and $x=\Delta^{m} y$. Then $y \in \mathrm{S}(0), x \in \mathrm{S}(m)$ and

$$
\begin{aligned}
\left|x_{n}\right| & =\left|\Delta^{m} y_{n}\right|=\left|\sum_{i=0}^{m}(-1)^{m+i}\left(\begin{array}{c}
m \\
i
\end{array}\right) y_{n+i}\right| \\
& =\left|\sum_{i=0}^{m}(-1)^{m+i}\left(\begin{array}{c}
m \\
i
\end{array}\right)(-1)^{n+i}(n+i)^{-\delta}\right|=\left|(-1)^{m+n} \sum_{i=0}^{m}\left(\begin{array}{c}
m \\
i
\end{array}\right)(n+i)^{-\delta}\right| \geq n^{-\delta} .
\end{aligned}
$$

Hence $n^{\varepsilon}\left|x_{n}\right| \geq n^{\varepsilon-\delta} \rightarrow \infty$ and $x \in \mathrm{S}(m) \backslash \mathrm{O}\left(n^{-\varepsilon}\right)$.

\section{Absolute summable sequences}

In this section we introduce the spaces $\mathrm{A}(m)$ of absolutely $m$-times summable sequences. We establish the relationships between $\mathrm{A}(m)$ and the spaces $\mathrm{o}\left(n^{s}\right)$ and $\mathrm{O}\left(n^{s}\right)$. There exist many tests for the absolute convergence of series. Most of them may be extended to the case $\mathrm{A}(m)$. For example we present five of them in Lemmas 4.3-4.7. At the end of the section, using all possible $\Delta^{p} \mathrm{~A}(m)$ and $r^{p} \mathrm{~A}(m)$ we obtain an infinite stratification of the set $S(0) \backslash A(\infty)$.

For $m \in \mathbb{N}(0)$ we define the set $\mathrm{A}(m)$ by

$$
\mathrm{A}(m)=\{a \in \mathrm{SQ}:|a| \in \mathrm{S}(m)\} .
$$

Moreover, let

$$
\mathrm{A}(\infty)=\bigcap_{k=0}^{\infty} \mathrm{A}(k), \quad \mathrm{o}\left(n^{-\infty}\right)=\bigcap_{s \in \mathbb{R}} \mathrm{o}\left(n^{s}\right), \quad \operatorname{Fin}=\bigcup_{p=1}^{\infty} \operatorname{Fin}(p) .
$$

Remark 4.1 Note that $\mathrm{A}(0)=\mathrm{S}(0)$ and the condition $a \in \mathrm{A}(1)$ is equivalent to the absolute convergence of the series $\sum_{n=1}^{\infty} a_{n}$. Moreover, $\mathrm{A}(m)$ is a linear subspace of $\mathrm{S}(m)$ for any $m \in \mathbb{N}(0)$. Note also that if $p \in \mathbb{N}$ and $\lambda \in(-1,1)$, then

$$
\operatorname{Fin}(p) \subset \operatorname{Fin} \subset \mathrm{o}\left(\lambda^{n}\right) \subset \mathrm{A}(\infty)
$$

Lemma 4.1 Assume $a \in \mathrm{SQ}, m \in \mathbb{N}(0)$ and $s \in \mathbb{R}$. Then

(a) $a \in \mathrm{A}(m+1) \Leftrightarrow\left(n^{m} a_{n}\right) \in \mathrm{A}(1)$,

(b) $a \in \mathrm{A}(m+1) \Leftrightarrow\left(n a_{n}\right) \in \mathrm{A}(m)$

(c) $\left(n^{s}\right) \in \mathrm{A}(m) \Leftrightarrow s<-m$.

Proof Assertion (a) is a consequence of Lemma 3.1(03), (b) is a consequence of (a). Assertion (c) follows from (b) and from the fact that the condition $\left(n^{s}\right) \in \mathrm{A}(1)$ is equivalent to the condition $s<-1$.

Example 4.1 Let $m \in \mathbb{N}(0)$. If we define a sequence $x$ by

$$
x_{n}=\frac{1}{n^{m} \log n},
$$

then, using Lemma 4.1(a), we obtain $x \in \mathrm{o}\left(n^{-m}\right) \backslash \mathrm{A}(m)$. 
Example 4.2 Assume $m \in \mathbb{N}$ and $\varepsilon>0$. Choose $p \in \mathbb{N}$ such that $p^{-1}<\varepsilon$. Let

$$
A=\left\{k^{2 p}: k \in \mathbb{N}\right\}, \quad B=\mathbb{N} \backslash A, \quad a_{n}= \begin{cases}n^{-p^{-1}} & \text { for } n \in A, \\ n^{-2} & \text { for } n \in B .\end{cases}
$$

Then

$$
\sum_{n \in A} a_{n}=\sum_{k=1}^{\infty}\left(k^{2 p}\right)^{-p^{-1}}=\sum_{k=1}^{\infty} k^{-2}<\infty, \quad \sum_{n \in B} a_{n} \leq \sum_{n=1}^{\infty} n^{-2}<\infty
$$

and $n^{\varepsilon} a_{n}=n^{\varepsilon-p^{-1}}$ for $n \in A$. Hence $\limsup _{n \rightarrow \infty} n^{\varepsilon} a_{n}=\infty$ and we obtain $a \in \mathrm{A}(1) \backslash \mathrm{O}\left(n^{-\varepsilon}\right)$. Let $b_{n}=n^{1-m} a_{n}$. Then, by Lemma 4.1(a),

$$
b \in \mathrm{A}(m) \backslash \mathrm{O}\left(n^{-m+1-\varepsilon}\right) .
$$

Remark 4.2 Note that for real $s, t$ the conditions $\mathrm{o}\left(n^{s}\right) \subset \mathrm{o}\left(n^{t}\right)$ and $\mathrm{O}\left(n^{s}\right) \subset \mathrm{O}\left(n^{t}\right)$ are equivalent to the condition $s \leq t$.

Lemma 4.2 Assume $m \in \mathbb{N}, \varepsilon>0$ and $s \in \mathbb{R}$. Then

(a) $\mathrm{O}\left(n^{-m-\varepsilon}\right) \subset \mathrm{A}(m) \subset \mathrm{o}\left(n^{-m+1}\right)$,

(b) $\mathrm{o}\left(n^{s}\right) \subset \mathrm{A}(m) \Leftrightarrow s<-m$,

(c) $\mathrm{A}(m) \subset \mathrm{O}\left(n^{s}\right) \Leftrightarrow s \geq-m+1$,

(d) $\mathrm{A}(\infty)=\mathrm{o}\left(n^{-\infty}\right)$.

Proof Assertion (a) follows from Lemma 4.1, (b) is a consequence of (a) and Example 4.1, (c) is a consequence of (a) and Example 4.2. Let $m \in \mathbb{N}(0)$. If $s<-m$, then, by (b), o( $\left.n^{s}\right) \subset$ $\mathrm{A}(m)$. Hence $\mathrm{o}\left(n^{-\infty}\right) \subset \mathrm{A}(m)$ for any $m \in \mathbb{N}(0)$. Therefore

$$
\mathrm{o}\left(n^{-\infty}\right) \subset \mathrm{A}(\infty)
$$

Let $s \in \mathbb{R}$. Choose $m \in \mathbb{N}(0)$ such that $m>1-s$. If $a \in \mathrm{A}(m)$, then, by the convergence of the series $\sum_{n=1}^{\infty} n^{m-1}\left|a_{n}\right|$, we have $n^{m-1} a_{n}=\mathrm{o}(1)$ and so $a \in \mathrm{o}\left(n^{1-m}\right) \subset \mathrm{o}\left(n^{s}\right)$. Hence $\mathrm{A}(m) \subset$ $\mathrm{o}\left(n^{s}\right)$. Therefore $\mathrm{A}(\infty) \subset \mathrm{o}\left(n^{s}\right)$ for any $s \in \mathbb{R}$ and we obtain

$$
\mathrm{A}(\infty) \subset \mathrm{o}\left(n^{-\infty}\right)
$$

Lemma 4.3 (Comparison test) Assume $a, b \in \mathrm{SQ}$ and $m \in \mathbb{N}$. Then

(a) if $\left|a_{n}\right| \leq\left|b_{n}\right|$ for large $n$ and $b \in \mathrm{A}(m)$, then $a \in \mathrm{A}(m)$,

(b) if $\left|a_{n}\right| \geq\left|b_{n}\right|$ for large $n$ and $b \notin \mathrm{A}(m)$, then $a \notin \mathrm{A}(m)$,

(c) if $\limsup \left|a_{n} / b_{n}\right|<\infty$ and $b \in \mathrm{A}(m)$, then $a \in \mathrm{A}(m)$,

(d) if $\liminf \left|a_{n} / b_{n}\right|>0$ and $a \in \mathrm{A}(m)$, then $b \in \mathrm{A}(m)$,

(e) if $\left|a_{n+1} / a_{n}\right| \leq\left|b_{n+1} / b_{n}\right|$ for large $n$ and $b \in \mathrm{A}(m)$, then $a \in \mathrm{A}(m)$.

Proof Assertion (a) follows from Lemma 3.1(13). Assertions (b), (c), (d), and (e) are consequences of (a). 
Lemma 4.4 (Generalized logarithmic test) Assume $a \in \mathrm{SQ}, m \in \mathbb{N}$ and

$$
u_{n}=\frac{\log \left|a_{n}\right|}{\log n} .
$$

\section{Then}

(a) if $\lim \sup u_{n}<-m$, then $a \in \mathrm{A}(m)$,

(b) if $u_{n} \geq-m$ for large $n$, then $a \notin \mathrm{A}(m)$,

(c) if liminf $u_{n}>-m$, then $a \notin \mathrm{A}(m)$,

(d) if $\lim u_{n}=-\infty$, then $a \in \mathrm{A}(\infty)$.

Proof If $\lim \sup u_{n}<-m$, then there exists a number $s<-m$ such that $u_{n}<s$ for large $n$. Then $\left|a_{n}\right| \leq n^{s}$ for large $n$. Hence, using Lemma 4.1 and Lemma 4.3, we obtain (a). If $u_{n} \geq$ $-m$ for large $n$, then $\left|a_{n}\right| \geq n^{-m}$ for large $n$ and (b) follows from Lemma 4.3 and the fact that $\left(n^{-m}\right) \notin \mathrm{A}(m)$. Assertion (c) follows immediately from (b), and (d) is a consequence of (a).

Lemma 4.5 (Generalized Raabe test) Assume $a \in \mathrm{SQ}, m \in \mathbb{N}$ and

$$
u_{n}=n\left(\frac{\left|a_{n}\right|}{\left|a_{n+1}\right|}-1\right) \text {. }
$$

\section{Then}

(a) if $\liminf u_{n}>m$, then $a \in \mathrm{A}(m)$,

(b) if $u_{n} \leq m$ for large $n$, then $a \notin \mathrm{A}(m)$,

(c) if $\lim \sup u_{n}<m$, then $a \notin \mathrm{A}(m)$,

(d) if $\lim u_{n}=\infty$, then $a \in \mathrm{A}(\infty)$.

Proof For $m=1$ assertion (a) follows from the usual Raabe test. Assume it is true for certain $m \geq 1$ and $\liminf u_{n}>m+1$. Let

$$
b_{n}=n a_{n}, \quad w_{n}=n\left(\frac{\left|b_{n}\right|}{\left|b_{n+1}\right|}-1\right) .
$$

Then

$$
w_{n}=n\left(\frac{n\left|a_{n}\right|}{(n+1)\left|a_{n+1}\right|}-\frac{n+1}{n+1}\right)=\frac{n}{n+1}\left(\frac{n\left|a_{n}\right|}{\left|a_{n+1}\right|}-n-1\right)=\frac{n}{n+1}\left(u_{n}-1\right) .
$$

Hence $\liminf w_{n}=\liminf u_{n}-1>m$ and, by inductive hypothesis, $b \in \mathrm{A}(m)$. Hence, by Lemma 4.1(b), $a \in \mathrm{A}(m+1)$ and we obtain (a). Similarly we may obtain (b), by taking $b_{n}=n a_{n}$, using the usual Raabe test and Lemma 4.1(b). Assertion (c) follows from (b), and (d) is a consequence of (a).

Lemma 4.6 (Generalized Gauss test) Let $a \in \mathrm{SQ}, m \in \mathbb{N}, \alpha, \beta \in \mathbb{R}, s<-1$ and

$$
\frac{\left|a_{n}\right|}{\left|a_{n+1}\right|}=\alpha+\frac{\beta}{n}+\mathrm{O}\left(n^{s}\right)
$$

\section{Then}

(a) if $\alpha>\lambda>1$, then $a \in \mathrm{o}\left(\lambda^{n}\right)$, 
(b) if $\alpha<1$, then $a \notin \mathrm{o}(1)$,

(c) if $\alpha=1$ and $\beta>m$, then $a \in \mathrm{A}(m)$,

(d) if $\alpha=1$ and $\beta \leq m$, then $a \notin \mathrm{A}(m)$.

Proof Note that

$$
\lim _{n \rightarrow \infty} \frac{\left|a_{n}\right|}{\left|a_{n+1}\right|}=\alpha .
$$

Hence (a) and (b) follow from the d'Alembert ratio test. For $m=1$ assertions (c) and (d) follow from the usual Gauss test. Assume they are true for certain $m \geq 1$. Let $b_{n}=n a_{n}$, $u_{n}=1 /(n+1)$. Then

$$
\begin{aligned}
\frac{\left|b_{n}\right|}{\left|b_{n+1}\right|} & =\frac{n}{n+1} \frac{\left|a_{n}\right|}{\left|a_{n+1}\right|}=\left(1-u_{n}\right)\left(1+\frac{\beta}{n}+\mathrm{O}\left(n^{s}\right)\right) \\
& =1+\frac{\beta}{n}+\mathrm{O}\left(n^{s}\right)-\frac{1}{n+1}-\frac{\beta}{n(n+1)}-\frac{1}{n+1} \mathrm{O}\left(n^{s}\right) \\
& =1+\frac{\beta-1}{n}+\left(\frac{1}{n}-\frac{1}{n+1}\right)+\mathrm{O}\left(n^{s}\right)+\mathrm{O}\left(n^{-2}\right)+\mathrm{O}\left(n^{s-1}\right) \\
& =1+\frac{\beta-1}{n}+\mathrm{O}\left(n^{s^{\prime}}\right)
\end{aligned}
$$

for certain $s^{\prime}<-1$. If $\beta>m+1$, then $\beta-1>m$ and, by inductive hypothesis, $b \in \mathrm{A}(m)$. Similarly, if $\beta \leq m+1$, then $b \notin \mathrm{A}(m)$. Now, assertions (c) and (d) follow from Lemma 4.1(b).

Lemma 4.7 (Generalized Bertrand test) Assume $a \in \mathrm{SQ}, m \in \mathbb{N}$ and

$$
\frac{\left|a_{n}\right|}{\left|a_{n+1}\right|}=1+\frac{m}{n}+\frac{\lambda_{n}}{n \log n} .
$$

\section{Then}

(a) if $\liminf \lambda_{n}>1$, then $a \in \mathrm{A}(m)$,

(b) if $\lambda_{n} \leq 1$ for large $n$, then $a \notin \mathrm{A}(m)$,

(c) if $\limsup \lambda_{n}<1$, then $a \notin \mathrm{A}(m)$.

Proof Let $b_{n}=n a_{n}$. Then

$$
\begin{aligned}
\frac{\left|b_{n}\right|}{\left|b_{n+1}\right|} & =\frac{n}{n+1}\left(1+\frac{m}{n}+\frac{\lambda_{n}}{n \ln n}\right) \\
& =\frac{n}{n+1}+\frac{m}{n+1}+\frac{\lambda_{n}}{(n+1) \log n} \\
& =1+\frac{m-1}{n+1}+\left(\frac{n}{n+1}\right) \frac{\lambda_{n}}{n \log n} \\
& =1+\frac{m-1}{n}-c_{n}+\frac{z_{n} \lambda_{n}}{n \log n},
\end{aligned}
$$

where

$$
z_{n}=\frac{n}{n+1}, \quad c_{n}=\frac{m-1}{n}-\frac{m-1}{n+1}=\frac{m-1}{n(n+1)} .
$$


Let

$$
w_{n}=c_{n} n \log n=\frac{(m-1) \log n}{n+1}, \quad \tau_{n}=z_{n} \lambda_{n}-w_{n} .
$$

Then

$$
\lim w_{n}=0, \quad \lim z_{n}=1, \quad \liminf \tau_{n}=\liminf \lambda_{n}
$$

and

$$
\frac{\left|b_{n}\right|}{\left|b_{n+1}\right|}=1+\frac{m-1}{n}+\frac{\tau_{n}}{n \log n} .
$$

Moreover,

$$
w_{n} \geq 0, \quad 0<z_{n}<1, \quad \tau_{n} \leq \lambda_{n} .
$$

For $m=1$ assertion (a) follows from the classical Bertrand test. Assume it is true for certain $m-1 \geq 1$. Then using the inductive hypothesis, (8), and (7), we have $b \in \mathrm{A}(m-1)$. Hence, by Lemma 4.1, $a \in \mathrm{A}(m)$ and we obtain (a). Analogously, using (9), we obtain (b). Assertion (c) is a consequence of $(b)$. The proof is complete.

Remark 4.3 Computing $\lambda_{n}$ from (6) we have

$$
\lambda_{n}=\left(n\left(\frac{\left|a_{n}\right|}{\left|a_{n+1}\right|}-1\right)-m\right) \log n .
$$

Replacing (6) by (10) in Lemma 4.7 one can obtain another form of the Bertrand test.

Lemma 4.8 Assume $m \in \mathbb{N}$. Then

(a) $\Delta \mathrm{A}(m) \subset \mathrm{A}(m)$,

(b) $\mathrm{A}(m) \subset r \mathrm{~A}(m)$,

(c) $r \mathrm{~A}(m) \subset \mathrm{A}(m-1)$,

(d) $\mathrm{A}(m) \subset \Delta \mathrm{A}(m-1)$.

Proof For $y \in$ SQ let $\mathrm{E} y \in \mathrm{SQ}$ be defined by

$$
\mathrm{E} y(n)=y(n+1) .
$$

Let $x \in \mathrm{S}(m)$. By Lemma 3.1(09) there exists $y \in \mathrm{S}(0)$ such that $x=\Delta^{m} y$. Obviously $\mathrm{E} \Delta y=$ $\Delta \mathrm{E} y$ and, by induction, $\mathrm{E} \Delta^{m} y=\Delta^{m} \mathrm{E} y$. Moreover, $\mathrm{E} y \in \mathrm{S}(0)$. Hence

$$
\mathrm{E} x=\mathrm{E} \Delta^{m} y=\Delta^{m} \mathrm{E} y \in \mathrm{S}(m) .
$$

Now assume $x \in \mathrm{A}(m)$. Then $|x| \in \mathrm{S}(m)$ and $|\mathrm{E} x|=\mathrm{E}|x| \in \mathrm{S}(m)$. Hence

$$
0 \leq|\Delta x| \leq|\mathrm{E} x|+|x| \in \mathrm{S}(m) .
$$


By Lemma 3.1(13) we have $\Delta x \in \mathrm{A}(m)$. Hence

$$
\Delta \mathrm{A}(m) \subset \mathrm{A}(m) \quad \text { and } \quad \mathrm{A}(m)=r \Delta \mathrm{A}(m) \subset r \mathrm{~A}(m) .
$$

From $|x| \in \mathrm{S}(m)$ we have $r|x| \in \mathrm{S}(m-1)$. Moreover, $0 \leq|r x| \leq r|x|$. Hence $|r x| \in \mathrm{S}(m-1)$. Therefore $r x \in \mathrm{A}(m-1)$ and we obtain

$$
r \mathrm{~A}(m) \subset \mathrm{A}(m-1), \quad \mathrm{A}(m)=\Delta r \mathrm{~A}(m) \subset \Delta \mathrm{A}(m-1) .
$$

The proof is complete.

Example 4.3 Let $m \in \mathbb{N}, x_{n}=n^{-m}, y=\Delta x$. By Theorem 2.2 in [7], we have $y \in \mathrm{O}\left(n^{-m-1}\right)$. Hence, by Lemma 4.2, $y \in \mathrm{A}(m)$. On the other hand $x \notin \mathrm{A}(m)$ and we obtain $y=\Delta x \notin$ $\Delta \mathrm{A}(m)$. Therefore $y \in \mathrm{A}(m) \backslash \Delta \mathrm{A}(m)$.

Example 4.4 Let $m \in \mathbb{N}, s \in(m, m+1], x_{n}=(-1)^{n} n^{-s}$. Then, by Lemma 4.1, $x \in \mathrm{A}(m) \backslash$ $\mathrm{A}(m+1)$. Assume $x \in r \mathrm{~A}(m+1)$. Choose $y \in \mathrm{A}(m+1)$ such that $x=r y$. Then, using Lemma 3.1(07), we obtain $\Delta x=\Delta r y=-y \in \mathrm{A}(m+1)$. Since $x$ is alternating we have $|\Delta x| \geq|x|$. Hence, by Lemma 4.3, $y=\Delta x \notin \mathrm{A}(m+1)$. This contradiction shows that $x \in \mathrm{A}(m) \backslash r \mathrm{~A}(m+1)$.

Remark 4.4 Using Lemma 4.8 we obtain the following infinite diagram, where arrows denote inclusions:

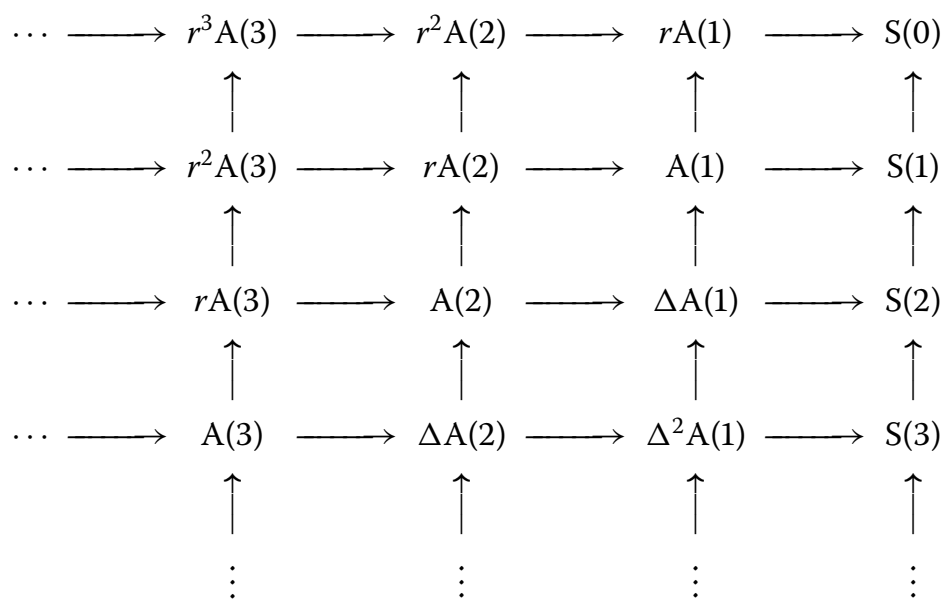

Using Remark 3.2 and Example 4.3 we can see that any vertical arrow represents a proper inclusion. Analogously, using Remark 3.2 and Example 4.4 we can see that any horizontal arrow represents a proper inclusion.

Remark 4.5 Introducing new notation

$$
\begin{array}{ll}
\mathrm{S}_{k}^{k+p}=\Delta^{p} \mathrm{~A}(k), & \mathrm{S}_{k}^{k-m}=r^{m} \mathrm{~A}(k), \\
\mathrm{S}_{k}^{\infty}=\bigcap_{n=0}^{\infty} \mathrm{S}_{k}^{n}, & \mathrm{~S}_{\infty}^{n}=\bigcap_{k=0}^{\infty} \mathrm{S}_{k}^{n}, \quad \mathrm{~S}_{\infty}^{\infty}=\bigcap_{k=0}^{\infty} \mathrm{S}_{k}^{k}
\end{array}
$$


for $k, p \in \mathbb{N}(0)$ and $m \in \mathbb{N}(0, k)$ we can extend the diagram from Remark 4.4 in the following way:

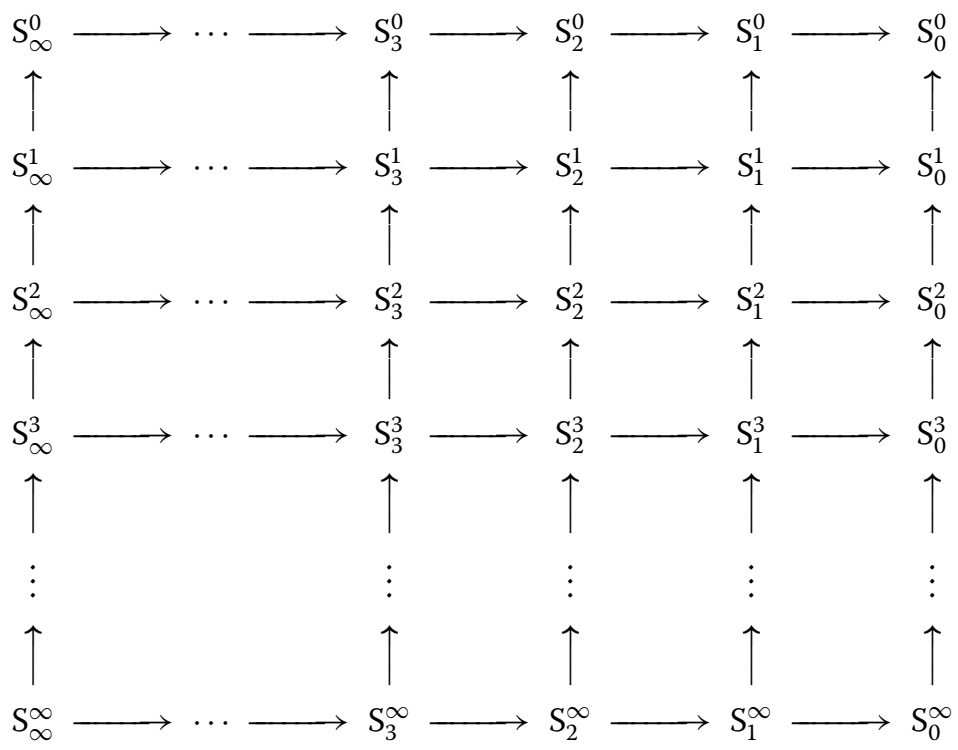

Note that if $k, n, p \in \mathbb{N}(0)$ and $m \in \mathbb{N}(0, n)$, then

$$
\begin{aligned}
& \Delta^{p} S_{k}^{n}=\mathrm{S}_{k}^{n+p}, \quad r^{m} \mathrm{~S}_{k}^{n}=\mathrm{S}_{k}^{n-m}, \quad \Delta^{p} \mathrm{~S}_{\infty}^{n}=\mathrm{S}_{\infty}^{n+p}, \quad r^{m} \mathrm{~S}_{\infty}^{n}=\mathrm{S}_{\infty}^{n-m}, \\
& \Delta^{m} \mathrm{~S}_{k}^{\infty}=\mathrm{S}_{k}^{\infty}=r^{m} \mathrm{~S}_{k}^{\infty}, \quad \Delta^{m} \mathrm{~S}_{\infty}^{\infty}=\mathrm{S}_{\infty}^{\infty}=r^{m} \mathrm{~S}_{\infty}^{\infty} .
\end{aligned}
$$

Moreover,

$$
\mathrm{S}_{\infty}^{\infty}=\mathrm{A}(\infty)=\mathrm{o}\left(n^{-\infty}\right)
$$

\section{Approximative solutions of difference equations}

In this section we present some applications of our previous results in the study of asymptotic properties of solutions of difference equations. We use the spaces $\mathrm{A}(p)$ to measure the 'degree of approximation'.

Assume $m \in \mathbb{N}, a, b \in \mathrm{SQ}, f: \mathbb{R} \rightarrow \mathbb{R}, \sigma: \mathbb{N} \rightarrow \mathbb{N}$ and $\lim _{n \rightarrow \infty} \sigma(n)=\infty$. We consider the equation

$$
\Delta^{m} x_{n}=a_{n} f\left(x_{\sigma(n)}\right)+b_{n} .
$$

By a solution of (E) we mean a sequence $x: \mathbb{N} \rightarrow \mathbb{R}$ satisfying (E) for all large $n$. Note that the assumption $\sigma: \mathbb{N} \rightarrow \mathbb{N}$ does not exclude the case of equations with delayed argument. For example we may define $\sigma(n)=n-5$ for $n>5$ and $\sigma(n)=1$ for $n \leq 5$.

In Theorem 5.1, using fixed point theorems and the iterated remainder operator, we establish conditions under which there exist solutions of (E) with prescribed asymptotic behavior. In Theorem 5.2 we show that in many cases some assumptions of Theorem 5.1 are necessary. In Theorem 5.3 we establish conditions under which all $f$-bounded solutions of (E) are asymptotically polynomial.

In the proof of our first theorem we will use the Schauder fixed point theorem and the following version of the Knaster-Tarski fixed point theorem. 
Lemma 5.1 If $X$ is a complete partially ordered set and a map $T: X \rightarrow X$ is nondecreasing then there exists $x_{0} \in X$ such that $T\left(x_{0}\right)=x_{0}$.

A simple proof of this result can be found in [20] (or in [19]).

Theorem 5.1 Assume $p \in \mathbb{N}(0), a \in \mathrm{A}(m+p), y \in \mathrm{SQ}, \Delta^{m} y=b, f$ is bounded on some uniform neighborhood $U$ of the set $y(\mathbb{N})$ and one of the following conditions is satisfied:

(a) $f$ is nondecreasing on $U$ and $(-1)^{m} a_{n} \geq 0$ for large $n$,

(b) $f$ is nonincreasing on $U$ and $(-1)^{m} a_{n} \leq 0$ for large $n$,

(c) $f$ is continuous on $U$.

Then there exists a solution $x$ of (E) such that

$x \in y+\mathrm{A}(p)$.

Proof For $x \in \mathrm{SQ}$ let $\bar{x}=f \circ x \circ \sigma$. Choose $c>0$ and $M>0$ such that

$$
\bigcup_{n=1}^{\infty}\left(y_{n}-c, y_{n}+c\right) \subset U, \quad|f(t)| \leq M \quad \text { for } t \in U .
$$

Let

$$
\rho=M r^{m}|a| .
$$

Choose numbers $k_{0}$ and $k$ such that $\rho_{n}<c$ for $n \geq k_{0}$ and $\sigma(n) \geq k_{0}$ for $n \geq k$. Let

$$
S=\left\{x \in \mathrm{SQ}:|x-y| \leq \rho \text { and } x_{n}=y_{n} \text { for } n<k\right\} .
$$

It is easy to see that $S$, with natural order defined by $x \leq z$ if $x_{n} \leq z_{n}$ for every $n \in \mathbb{N}$, is a complete partially ordered set. If $x \in S$ and $n \geq k$, then $\left|x_{\sigma(n)}-y_{\sigma(n)}\right|<c$. Hence

$$
x_{\sigma(n)} \in U
$$

for any $x \in S$ and $n \geq k$. If $x \in S$, then, by (11) and (12), the sequence $\bar{x}$ is bounded and so $a \bar{x} \in \mathrm{A}(m+p)$. We define an operator $H: S \rightarrow$ SQ by

$$
H(x)(n):= \begin{cases}y_{n} & \text { for } n<k, \\ y_{n}+(-1)^{m} r_{n}^{m}(a \bar{x}) & \text { for } n \geq k .\end{cases}
$$

If $n \geq k$, then, using Lemma 3.1(01) and Lemma 3.1(14), we obtain

$$
\left|H(x)(n)-y_{n}\right|=\left|r_{n}^{m}(a \bar{x})\right| \leq r_{n}^{m}|a \bar{x}| \leq M r_{n}^{m}|a|=\rho_{n} .
$$

Hence $H(S) \subset S$.

Assume now that the condition (a) is satisfied. We can assume that $(-1)^{m} a_{n} \geq 0$ for $n \geq k$. Let $x, z \in S$ and $x \leq z$. Since $f$ is nondecreasing on $U$ we have $f\left(x_{\sigma(n)}\right) \leq f\left(z_{\sigma(n)}\right)$ for $n \geq k$. Hence

$$
(-1)^{m} a_{n} f\left(x_{\sigma(n)}\right) \leq(-1)^{m} a_{n} f\left(z_{\sigma(n)}\right)
$$


for $n \geq k$. By Lemma 3.1(11), we have $H(x)(n) \leq H(z)(n)$ for $n \geq k$. Moreover, $H(x)(n)=y_{n}=$ $H(z)(n)$ for $n<k$. Hence $H(x) \leq H(z)$. Now, using the Knaster-Tarski fixed point theorem we obtain $x \in \mathrm{S}$ such that $H(x)=x$. Analogously if condition (b) is satisfied then $H(x)=x$ for certain $x \in \mathrm{S}$.

Assume (c) and let $d$ be a metric on $S$ defined by

$$
d(x, z)=\|x-z\|=\sup _{n \in \mathbb{N}}\left|x_{n}-z_{n}\right|
$$

Let BS denote the Banach space of all bounded sequences $x \in \mathrm{SQ}$ with the norm $\|x\|=$ $\sup _{n \in \mathbb{N}}\left|x_{n}\right|$ and let

$$
T=\left\{x \in \mathrm{BS}:|x| \leq \rho \text { and } x_{n}=0 \text { for } n<k\right\} .
$$

It is easy to see that $T$ is a convex and closed subset of BS. Choose an $\varepsilon>0$. Then there exists $q \in \mathbb{N}$ such that $\rho_{n}<\varepsilon$ for $n \geq q$. For $n=1, \ldots, q$ let $G_{n}$ denote a finite $\varepsilon$-net for the interval $\left[-\rho_{n}, \rho_{n}\right]$ and let

$$
G=\left\{x \in T: x_{n} \in G_{n} \text { for } n \leq q \text { and } x_{n}=0 \text { for } n>q\right\} .
$$

Then $G$ is a finite $\varepsilon$-net for $T$. Hence $T$ is a complete and totally bounded metric space and so, $T$ is compact. Hence $T$ is a convex and compact subset of the Banach space BS and, by the Schauder fixed point theorem, any continuous map $T \rightarrow T$ has a fixed point. Let $F: T \rightarrow S$ be a map given by $F(x)(n)=x_{n}+y_{n}$. Then $F$ is an isometry of $T$ onto $S$. Assume $U: S \rightarrow S$ is a continuous map and let $W=F^{-1} \circ U \circ F$. Then $W: T \rightarrow T$ is continuous and there exists a point $z \in T$ such that $W z=z$. Let $x=F z$. Then

$$
x=F z=F W z=F F^{-1} U F z=U x .
$$

Hence any continuous map $U: S \rightarrow S$ has a fixed point. Let $\varepsilon>0$. Choose $q \in \mathbb{N}(k)$ and $\alpha>0$ such that

$$
M \sum_{m=q}^{\infty} n^{m-1}\left|a_{n}\right|<\varepsilon \quad \text { and } \quad \alpha \sum_{n=k}^{q} n^{m-1}\left|a_{n}\right|<\varepsilon .
$$

Let

$$
W=\bigcup_{n=k}^{q}\left[y_{n}-\rho_{n}, y_{n}+\rho_{n}\right]
$$

Then $W \subset U$. By compactness of $W, f$ is uniformly continuous on $W$. Hence there exists $\delta>0$ such that if $s, t \in W$ and $|s-t|<\delta$, then $|f(s)-f(t)|<\alpha$. Assume $x, z \in S, d(x, z)<\delta$. Let $u=\bar{x}-\bar{z}$. Then

$$
\begin{aligned}
d(H x, H z) & =\sup _{n \geq k}\left|r_{n}^{m}(a \bar{x})-r_{n}^{m}(a \bar{z})\right| \\
& =\sup _{n \geq k}\left|r_{n}^{m}(a u)\right| \leq \sup _{n \geq k} r_{n}^{m}|a u|=r_{p}^{m}|a u|
\end{aligned}
$$




$$
\begin{aligned}
& \leq \sum_{n=k}^{q} n^{m-1}\left|a_{n} u_{n}\right|+\sum_{n=q}^{\infty} n^{m-1}\left|a_{n} u_{n}\right| \\
& \leq \alpha \sum_{n=k}^{q} n^{m-1}\left|a_{n}\right|+2 M \sum_{n=q}^{\infty} n^{m-1}\left|a_{n}\right|<3 \varepsilon .
\end{aligned}
$$

Hence $H$ is continuous and there exists $x \in \mathrm{S}$ such that $H(x)=x$. Then

$$
x_{n}=H(x)(n)=y_{n}+(-1)^{m} r_{n}^{m}(a \bar{x})
$$

for $n \geq k$. Hence

$$
x-\left(y+(-1)^{m} r^{m}(a \bar{x})\right) \in \operatorname{Fin}(k) .
$$

Since the sequence $\bar{x}$ is bounded, we have $a \bar{x} \in \mathrm{O}(a)$. Hence

$$
x \in y+r^{m} \mathrm{O}(a)+\operatorname{Fin}(k) \subset y+\mathrm{A}(p) .
$$

Moreover, for $n \geq k$, by Lemma 3.1(07), we have

$$
\Delta^{m} x_{n}=\Delta^{m} y_{n}+(-1)^{m} \Delta^{m} r_{n}^{m}(a \bar{x})=b_{n}+a_{n} f\left(x_{\sigma(n)}\right) .
$$

Hence $x$ is a solution of (E). The proof is complete.

Remark 5.1 The conclusion $x \in y+\mathrm{A}(p)$ of Theorem 5.1 may be written in the form

$$
y \in x+\mathrm{A}(p) .
$$

We can say that $y$ is an approximative solution of (E) with 'degree of approximation' $\mathrm{A}(p)$.

Remark 5.2 Using Lemma 4.5 we can replace the assumption $a \in \mathrm{A}(m+p)$ of Theorem 5.1 by

$$
\liminf n\left(\frac{\left|a_{n}\right|}{\left|a_{n+1}\right|}-1\right)>m+p
$$

Analogously, the conclusion of this theorem may be replaced: there exist a solution $x$ of (E) and a sequence $z \in \mathrm{SQ}$ such that $x=y+z$ and

$$
\lim \sup n\left(\frac{\left|z_{n}\right|}{\left|z_{n+1}\right|}-1\right) \geq p
$$

Similarly, using other tests, we can obtain many formulations of this theorem.

Theorem 5.2 Assume $q \in \mathbb{N}, p \in \mathbb{N}(0), \varepsilon>0, s \in(-\infty,-p), a, b, y \in \mathrm{SQ}, \Delta^{m} y=b, a$ is nonoscillatory, there exists a uniform neighborhood $U$ of the set $y(\mathbb{N}(q))$ such that $f \mid U \geq \varepsilon$ or $f \mid U \leq-\varepsilon$, and one of the following conditions is satisfied:

(a) there exists a solution $x$ of (E) such that $x=y+o\left(n^{s}\right)$, 
(b) there exists a solution $x$ of (E) such that $x \in y+\mathrm{S}(p)$.

Then $a \in \mathrm{A}(m+p)$.

Proof By Lemma 4.2 we have $\mathrm{o}\left(n^{s}\right) \subset \mathrm{A}(p) \subset \mathrm{S}(p)$. Hence, there exists a solution $x$ of (E) and a sequence $z \in \mathrm{S}(p)$ such that $x=y+z$. Let $\bar{x}=f \circ x \circ \sigma$. For large $n$ we have

$$
a_{n} \bar{x}_{n}+b_{n}=a_{n} f\left(x_{\sigma(n)}\right)+b_{n}=\Delta^{m} x_{n}=\Delta^{m} y_{n}+\Delta^{m} z_{n}=b_{n}+\Delta^{m} z_{n} .
$$

By Lemma 3.1(10), $\Delta^{m} z \in \mathrm{S}(m+p)$. Hence, by Lemma 3.1(12), we have $a \bar{x} \in \mathrm{S}(m+p)$. Since $z_{n}=\mathrm{o}(1)$, we have $x_{\sigma(n)} \in U$ for large $n$. Hence the sequence $a \bar{x}$ is nonoscillatory and we obtain $|a \bar{x}| \in \mathrm{S}(m+p)$, which means $a \bar{x} \in \mathrm{A}(m+p)$. Moreover,

$$
\left|a_{n}\right|=\left|a_{n} \bar{x}_{n}\right|\left|\bar{x}_{n}\right|^{-1} \leq\left|a_{n} \bar{x}_{n}\right| \varepsilon^{-1}
$$

for large $n$. Hence, by Lemma 3.1(04), $a \in \mathrm{A}(m+p)$. The proof is complete.

Remark 5.3 Theorem 5.2 extends Theorem 1 of [8] and parts of Theorems 6 and 7 of [6].

Lemma 5.2 Assume $m \in \mathbb{N}, p \in \mathbb{N}(0), x \in \mathrm{SQ}$ and $\Delta^{m} x \in \mathrm{A}(m+p)$. Then

$$
x \in \operatorname{Pol}(m-1)+\mathrm{A}(p) .
$$

Proof Using Lemma 4.8(c) we obtain

$$
r^{m} \mathrm{~A}(m+p) \subset \mathrm{A}(p) .
$$

Let $z=(-1)^{m} r^{m} \Delta^{m} x$. Then $\Delta^{m} z=\Delta^{m}(-1)^{m} r^{m} \Delta^{m} x=\Delta^{m} x$. Hence

$$
x-z \in \operatorname{Ker} \Delta^{m}=\operatorname{Pol}(m-1) .
$$

By (13) we have $z \in \mathrm{A}(p)$. Hence

$$
x=(x-z)+z \in \operatorname{Pol}(m-1)+\mathrm{A}(p) .
$$

Theorem 5.3 Assume $p \in \mathbb{N}(0), a, b \in \mathrm{A}(m+p)$ and $x$ is an $f$-bounded solution of (E).

Then

$$
x \in \operatorname{Pol}(m-1)+\mathrm{A}(p) .
$$

Proof The sequence $\bar{x}=f \circ x \circ \sigma$ is bounded. Hence $a \bar{x} \in \mathrm{O}(a)$ and we obtain $\Delta^{m} x \in$ $\mathrm{O}(|a|+|b|)$. Therefore $\Delta^{m} x \in \mathrm{A}(m+p)$ and, by Lemma 5.2, we have

$$
x \in \operatorname{Pol}(m-1)+\mathrm{A}(p) .
$$

\section{Competing interests}

The author declares that he has no competing interests. 


\section{References}

1. Huang, $X, X u, Z$ : Nonoscillatory solutions of certain higher order neutral difference equations. Southeast Asian Bull. Math. 32, 445-458 (2008)

2. Li, WT, Fan, XL, Zhong, CK: Unbounded positive solutions of higher-order difference equations with singular nonlinear term. Comput. Math. Appl. 39(3-4), 177-184 (2000)

3. Liu, M, Guo, Z: Solvability of a higher-order nonlinear neutral delay difference equation. Adv. Differ. Equ. 2010, Article ID 767620 (2010)

4. Migda, M, Migda, J: On the asymptotic behavior of solutions of higher order nonlinear difference equations. Nonlinear Anal. 47, 4687-4695 (2001)

5. Zhu, ZQ, Wang, GQ, Cheng, SS: A classification scheme for nonoscillatory solutions of a higher order neutral difference equation. Adv. Differ. Equ. 2006, Article ID 47654 (2006)

6. Migda, J: Asymptotic properties of solutions of nonautonomous difference equations. Arch. Math. 46, 1-11 (2010)

7. Migda, J: Asymptotically polynomial solutions of difference equations. Adv. Differ. Equ. 2013, 92 (2013)

8. Drozdowicz, A, Popenda, J: Asymptotic behavior of the solutions of the second order difference equations. Proc. Am. Math. Soc. 99(1), 135-140 (1987)

9. Drozdowicz, A, Popenda, J: Asymptotic behavior of the solutions of an $n$th order difference equations. Ann. Soc. Math. Pol., Comment. Math. XXIX, 161-168 (1990)

10. Gleska, A, Werbowski, J: Comparison theorems for the asymptotic behavior of solutions of nonlinear difference equations. J. Math. Anal. Appl. 226(2), 456-465 (1998)

11. Popenda, J: Asymptotic properties of solutions of difference equations. Proc. Indian Acad. Sci. Math. Sci. 95(2), 141-153 (1986)

12. Popenda, J, Schmeidel, E: On the asymptotic behaviour of nonhomogeneous linear difference equations. Indian J. Pure Appl. Math. 28(3), 319-327 (1997)

13. Diblík, J: A criterion of asymptotic convergence for a class of nonlinear differential equations with delay. Nonlinear Anal. 47(6), 4095-4106 (2001)

14. Ehrnstrom, M: Linear asymptotic behaviour of second order ordinary differential equations. Glasg. Math. J. 49, 105-120 (2007)

15. Lipovan, $\mathrm{O}$ : On the asymptotic behavior of the solutions to a class of second order nonlinear differential equations. Glasg. Math. J. 45, 179-187 (2003)

16. Mingarelli, AB, Sadarangani, K: Asymptotic solutions of forced nonlinear second order differential equations and their extensions. Electron. J. Differ. Equ. 2007, 40 (2007)

17. Mustafa, OG, Rogovchenko, YV: Global existence of solutions with prescribed asymptotic behavior for second-order nonlinear differential equations. Nonlinear Anal. 51, 339-368 (2002)

18. Philos, CG, Purnaras, IK, Tsamatos, PC: Asymptotic to polynomials solutions for nonlinear differential equations. Nonlinear Anal. 59, 1157-1179 (2004)

19. Migda, J: Approximative solutions of difference equations. Electron. J. Qual. Theory Differ. Equ. 2014, 13 (2014)

20. Birkhoff, G: Lattice Theory. American Mathematical Society Colloquium Publications, vol. XXV (1940); 3rd edn. (1967)

10.1186/1687-1847-2014-189

Cite this article as: Migda: Iterated remainder operator, tests for multiple convergence of series, and solutions of difference equations. Advances in Difference Equations 2014, 2014:189

\section{Submit your manuscript to a SpringerOpen ${ }^{\circ}$ journal and benefit from:}

- Convenient online submission

Rigorous peer review

- Immediate publication on acceptance

Open access: articles freely available online

- High visibility within the field

- Retaining the copyright to your article 